Images du travail, travail des images

$9 \mid 2020$

L'apprentissage et l'enseignement professionnel en images

\title{
La télévision et l'enseignement professionnel : représentation de la massification scolaire et changement de paradigme socio-économique (1977-1987)
}

Television and vocational education: representation of school massification and socio-economic paradigm shift (1977-1987)

Jérôme Krop

\section{OpenEdition}

Journals

Édition électronique

URL : http://journals.openedition.org/itti/429

DOI : $10.4000 /$ itti. 429

Éditeur

Université de Poitiers

Référence électronique

Jérôme Krop, « La télévision et l'enseignement professionnel : représentation de la massification scolaire et changement de paradigme socio-économique (1977-1987) », Images du travail, travail des images [En ligne], 9 | 2020, mis en ligne le 01 septembre 2020, consulté le 14 avril 2021. URL : http:// journals.openedition.org/itti/429; DOI : https://doi.org/10.4000/itti.429

Ce document a été généré automatiquement le 14 avril 2021

Images du travail, travail des images 


\section{La télévision et l'enseignement professionnel : représentation de la massification scolaire et changement de paradigme socio- économique (1977-1987)}

Television and vocational education: representation of school massification and socio-economic paradigm shift (1977-1987)

Jérôme Krop

1 Alors même que l'essor de la télévision constitue un fait culturel majeur des années 1950 aux années 1980, la dynamique tant démographique, économique, sociale que culturelle de la France de l'après-guerre, qui se prolonge bien au-delà du milieu des années 1970 sur le plan scolaire, conduit à la mise en œuvre de politiques transformant profondément la physionomie du système éducatif. Du début de la Cinquième République au milieu des années 1990, qui marque un nouveau seuil en termes d'accès au baccalauréat général, cette transformation pérennise l'évolution du système scolaire vers une organisation en deux degrés successifs, notamment caractérisée par la scolarisation dans un même type d'établissement jusqu'à la classe de troisième (Gutierrez et Legris, 2016). Les réformes structurelles menées au début de la Vème République ont ainsi transformé la place de l'enseignement technique et de l'enseignement professionnel (Lembré, 2016), qui scolarisent une part croissante de la population scolaire orientée vers ces filières dans le cadre de leur scolarité obligatoire, l'enseignement professionnel et l'apprentissage se trouvant dès lors dans une position dominée dans le système scolaire par rapport à l'enseignement général (Palheta, 2012).

2 Le recours aux archives télévisuelles pour étudier les images de la massification scolaire, et en l'occurrence celles de l'enseignement professionnel, se justifie par la place croissante qu'occupe la télévision à cette époque dans la structuration des représentations sociales (Poels, 2015). En effet, la décennie de la fin des années 1970 à la 
fin des années 1980 constitue l'apogée de la domination des grandes chaînes de télévision hertzienne sur le champ médiatique, en l'absence d'une réelle concurrence qui n'apparaît que bien plus tard avec l'essor de la télévision satellitaire, des chaînes de la TNT et d'internet. La télévision acquiert donc à cette époque une forte légitimité à énoncer aux yeux des téléspectateurs la réalité du monde social, à travers les propos et les images qu'elle diffuse auprès d'un public croissant. En 1974, 85 \% des foyers sont équipés, soit quatorze millions de récepteurs (dix millions en 1969, un million seulement en 1958) (Sauvage M. et Veyrat-Masson I., 2012). L'offre de programme s'accroît mais TF1 et Antenne 2 rassemblent 80 \% de l'offre, FR3 ne diffusant que trois ou quatre heures par jour jusqu'en 1983. À cette date, Antenne 2 devance la première chaîne mais, en 1987, TF1, récemment privatisée, a retrouvé sa prédominance avec $41,5 \%$ d'audience, contre 35,1 pour sa cadette.

3 Au cours de cette période, la durée quotidienne d'écoute augmente fortement. Entre 1975 et 1985, le temps de loisir est passé de 3 h30 par jour à 4 heures. Les 30 minutes supplémentaires de loisir sont presque exclusivement transférées sur l'écoute de la télévision (Gaillard, 2012). Dans les années 1970 et 1980, la télévision ne s'est pas encore banalisée et des émissions assez longues, documentaires ou émissions de plateau, bénéficient probablement d'une attention plus soutenue des téléspectateurs avant la généralisation du zapping. Les documentaires et émissions de plateau diffusés à cette époque représentent donc une source que l'historien ne peut ignorer, même si les données disponibles sur la réception du discours télévisuels par les téléspectateurs sont très lacunaires (Poels, 2015).

4 Pour tenter une première analyse de l'évolution des représentations télévisuelles du travail des élèves et des enseignants de l'enseignement professionnel et de l'enseignement technique, nous nous proposons de mener une étude comparative autour de deux temps forts permettant d'appréhender comment la transformation du système scolaire mais aussi les mutations du contexte politique, économique et social et la reconfiguration du champ médiatique influencent ces représentations. L'émission $L a$ Réforme de l'éducation nationale intervient à un moment essentiel dans l'histoire de la transformation du système scolaire et de la place de l'enseignement professionnel en son sein. Pendant quatre semaines, du 17 mai au 7 juin 1977, chaque mardi soir, les téléspectateurs peuvent voir sur la première chaîne de télévision publique un documentaire dédié à la réforme issue du vote de la loi Haby du 11 juillet 1975, qui doit entrer en application à la rentrée en septembre. Issu d'une coproduction associant TF1 et l'Éducation nationale par l'intermédiaire du Centre national de documentation pédagogique (CNDP), ce cycle d'émissions, constitué de quatre documentaires de plus de 50 minutes diffusés à $21 \mathrm{~h} 30$, s'intéresse successivement aux écoles maternelles et élémentaires, aux collèges, aux lycées et à l'enseignement professionnel ${ }^{1}$. Il articule une description qui se veut objective de la situation du système éducatif et une présentation des principales dispositions de la réforme en cours. Aussi, des séquences tournées dans des établissements scolaires alternent-elles avec la mise en images de la présentation des dispositions de la réforme par de hauts fonctionnaires de l'Éducation nationale.

Le second temps fort est constitué par une émission de plateau réalisée dix ans plus tard sur la même chaîne, TF1, au lendemain de sa privatisation. Création nouvelle de la grille de programme de la rentrée 1987, l'émission Médiations, diffusée mensuellement jusqu'en 1992 en seconde partie de soirée, est l'archétype des émissions de débat des années 1980 (Rouquette, 2002). Elle aborde les problématiques scolaires dès le troisième 
numéro à propos du second degré sous le titre « l'échec scolaire $»^{2}$. Une décennie après le début de la mise en œuvre du principe du collège unique, deux ans après la proclamation de l'objectif fixé à 80 \% d'une classe d'âge au niveau du baccalauréat et la création des baccalauréats professionnels, Médiations accorde, comme La Réforme de l'éducation nationale, une place majeure à la question de l'orientation des élèves et à celle de l'enseignement professionnel ${ }^{3}$.

La physionomie du discours télévisuel est profondément transformée en une décennie, passant d'une communication institutionnelle très contrôlée par les pouvoirs publics à une forme de mise en scène tendant à faire de la télévision une instance du débat démocratique, alors même que les grands médias audiovisuels commencent à faire l'objet d'une appropriation économique par des capitaux privés. Aussi, ces sources doivent-elles être soumises à une analyse mettant en relation les montages des images filmées, qui peuvent s'apparenter au langage cinématographique, et les propos tenus directement par les auteurs ou par les intervenants (personnes interrogées dans le cadre d'une enquête journalistique ou invitées à débattre en plateau) (Jost, 2007).

7 Les images de l'enseignement professionnel, et tout particulièrement celles qui donnent à voir les élèves et les professeurs au travail, ne peuvent être dissociées du discours sur l'enseignement professionnel auquel elles participent, y compris dans sa dimension performative. D'abord au service d'un discours institutionnel destiné à valoriser l'orientation vers les filières professionnelles et techniques dans le cadre du collège unique naissant, elles participent dix ans plus tard à la promotion du rôle des entreprises dans ces formations, ce qui n'exclut pas une certaine cohérence dans l'évolution des schèmes idéologiques appliqués à ces enseignements.

\section{La Réforme de l'éducation nationale : un discours institutionnel de valorisation de l'enseignement professionnel}

\subsection{La place prépondérante de l'enseignement professionnel et technique}

8 La diffusion de La Réforme de l'éducation nationale marque une date importante dans l'histoire des représentations télévisuelles du système éducatif. En effet, dotés de moyens de tournage importants et du soutien, en tant que commanditaire, du ministère de l'Éducation nationale, les réalisateurs de cette série de documentaires ont $\mathrm{pu}$ filmer longuement dans tous les types d'établissements scolaires (Collège d'enseignement secondaire (CES), Collège d'enseignement général (CEG), Collège d'enseignement technique (CET), lycée technique, lycée général). Pour la première fois, les téléspectateurs se voient proposer une représentation d'ensemble $\mathrm{du}$ fonctionnement du système scolaire et des évolutions escomptées par les réformateurs. Les trois documentaires évoquant la transformation du second degré sont caractérisés par une volonté constante de promouvoir l'orientation vers des filières professionnelles et techniques comme perspective pour la majorité des élèves du collège unique. Les $\mathrm{VI}^{\mathrm{e}}$ (1971-1975) et VII ${ }^{e}$ plans (1976-1980), à la charnière desquels se situe le vote de la loi du 11 juillet 1975, privilégient les formations professionnelles et n'envisagent pas une augmentation substantielle du nombre de bacheliers généraux destinés à poursuivre des études supérieures (Chapoulie, 2010). Aussi, La Réforme de l'éducation nationale 
privilégie-t-elle clairement les orientations vers les sections professionnelles. L'apprentissage est peu évoqué : le propos de La Réforme de l'éducation nationale vise plutôt à promouvoir l'orientation en fin de troisième vers les lycées d'enseignement professionnel (LEP), nouvelle dénomination des collèges d'enseignement technique (CET), conformément à la perspective de création d'un tronc commun à tous les élèves de la $6^{\mathrm{e}}$ à la $3^{\mathrm{e}}$. L'orientation en fin de $5^{\mathrm{e}}$ pour la préparation d'un Certificat d'Aptitude Professionnel (CAP) en trois ans est présentée comme une orientation trop précoce et l'apprentissage semble réservé à certaines professions spécifiques, par exemple cuisinier, pour une petite minorité d'élèves ${ }^{4}$.

9 La Réforme de l'éducation nationale entend valoriser l'enseignement professionnel en l'associant étroitement, tant sur le plan de l'image elle-même que du commentaire, à l'enseignement technique, en mettant en scène une progressivité des représentations de l'un et de l'autre, reléguant ainsi l'enseignement général au second plan, y compris dans le documentaire dédié au lycée. Ainsi, alors qu'au cours de la première moitié de celui-ci, une séquence de 6'31" est constituée des témoignages d'élèves de la section $\mathrm{E}$ du lycée Jean Bart de Dunkerque, associant à la préparation d'un baccalauréat scientifique de type $\mathrm{C}$ une importante option technique ${ }^{5}$, les vingt dernières minutes sont exclusivement consacrées aux baccalauréats de technicien à travers la présentation de certaines réalisations pédagogiques de plusieurs établissements. Plusieurs d'entre eux sont explicitement présentés comme des lycées expérimentaux, dans lesquels seraient mises en œuvre des pratiques pédagogiques nouvellement promues à cette époque, par exemple les travaux de groupe en autonomie, qui ont vocation à essaimer par la suite. Aussi ne peuvent-ils être considérés comme représentatifs.

Les auteurs du documentaire diffusé la semaine suivante, consacré au LEP, souligne la partialité de leur point de vue en indiquant en conclusion qu'ils ont fait le choix de privilégier les formations industrielles formant de futurs ouvriers au détriment du secteur tertiaire et de la présence des jeunes filles, pratiquement absentes à l'écran alors qu'elles représentent pourtant $40 \%$ des élèves de l'enseignement technique ${ }^{6}$. Ce parti pris, déjà visible dans le film consacré au lycée, se retrouve donc dans le film sur le LEP. En introduction, Jean Pottier, inspecteur pédagogique régional, affirme, sur fond d'images en plan large d'un atelier de menuiserie, que l'objectif de l'enseignement professionnel est de former «des futurs travailleurs qui seront amenés dans un premier temps à être des ouvriers ", y compris contre les représentations dominantes faisant des emplois d'ouvriers d'industrie une position sociale peu enviable ${ }^{7}$. Cette présentation propose au téléspectateur d'assister au dialogue d'un enseignant avec des élèves du lycée d'Albi (Tarn) qui doivent usiner un support de perceuse pour introduire une réflexion sur la notion de culture technologique et sur la mise en responsabilité au travail propre aux conceptions pédagogiques de l'enseignement professionnel. Enfin, après une séquence au CET du bâtiment de Bagnolet Seine-Saint-Denis et une courte incursion dans un lycée hôtelier, la section de plasturgie du lycée de Haubourdin (Nord) constitue le pont d'orgue d'un discours institutionnel destiné à valoriser les formations industrielles censées associer excellence technique et modernité pédagogique. 


\subsection{La promotion d'une pédagogie de projet}

11 La pédagogie de projet est constamment mise en valeur par La réforme de l'éducation nationale comme facteur de motivation des élèves, ce qui l'assimile pédagogiquement aux méthodes actives popularisées depuis l'entre-deux-guerres par l'éducation nouvelle. La pédagogie de projet est présentée comme la déclinaison dans l'ensemble de l'enseignement professionnel et technique du «travail par thème » expérimenté dans les lycées techniques au niveau des terminales à partir de 1975 et qui vise à organiser le travail annuel des élèves autour d'une réalisation prise en compte à l'examen, remettant ainsi en cause le principe de prédominance des épreuves pratiques en temps limité dans les examens de l'enseignement technique (Pelpel, 2000).

Une place de choix est laissée à cette expérimentation rendant les élèves responsables d'une production technique, notamment avec l'exemple de la réalisation d'une unité industrielle de perçage par des élèves du lycée technique Paul Langevin de La Seynesur-Mer. Le documentaire articule des images tournées en cours mettant en scène l'enseignant exposant aux élèves le cahier des charges de la fabrication et la présentation technique par un élève des pièces qu'il a réalisées, l'ensemble étant associé sur un plan général aux images du fonctionnement de la machine transfert réalisée collectivement au cours de l'année scolaire.

Pour promouvoir cette rénovation des modalités d'examen, le proviseur du lycée technique Paul Langevin explique que :

«Le jury, en fin d'année, juge l'élève non pas sur un effort en temps limité mais sur un effort qui s'est étalé tout au long de l'année scolaire et qui a fait appel à toutes sortes de connaissances générales, scientifiques, technologiques et graphiques... Et les élèves que vous avez rencontrés dans l'établissement vous ont d'ailleurs dit qu'ils avaient eu le sentiment de réaliser quelque chose tout au long de l'année et de participer à une œuvre collective, et à une œuvre collective dont ils ont pu voir concrètement le résultat à la fin de l'année scolaire avant de passer le baccalauréat ${ }^{8}$.»

Inspiré du travail par thème, la pédagogie de projet est appelée à devenir la norme pédagogique de l'ensemble de l'enseignement professionnel et technique. Ainsi, les images des élèves et des professeurs du CAP gros œuvre du CET de Bagnolet, filmés en plan rapproché pendant qu'ils coulent la dalle en ciment du futur premier étage des nouvelles salles de leur propre collège, sont censées représenter à la fois le caractère pratique de cet enseignement et la dimension sociale d'un travail qui est d'abord la réalisation d'une équipe.

Le téléspectateur peut suivre plus particulièrement les différentes étapes de la réalisation de l'acrotère du toit, de la présentation par l'enseignant des préconisations $\mathrm{du}$ devis descriptif à la pose par les élèves de leur production sur le bâtiment. En salle de travaux dirigés, les élèves expérimentent le vibrage du béton destiné à en extraire les bulles d'air lors du coulage, l'enseignement technique étant explicitement comparé par le commentaire à un enseignement scientifique expérimental, bien que le rôle de l'enseignant soit sur les images manifestement prépondérant, tant en classe qu'en atelier, au détriment de la mise en activité autonome des élèves (fig. 1 et 2 ). 
Figure 1 : expérimentation de la vibration du béton au CET de Bagnolet

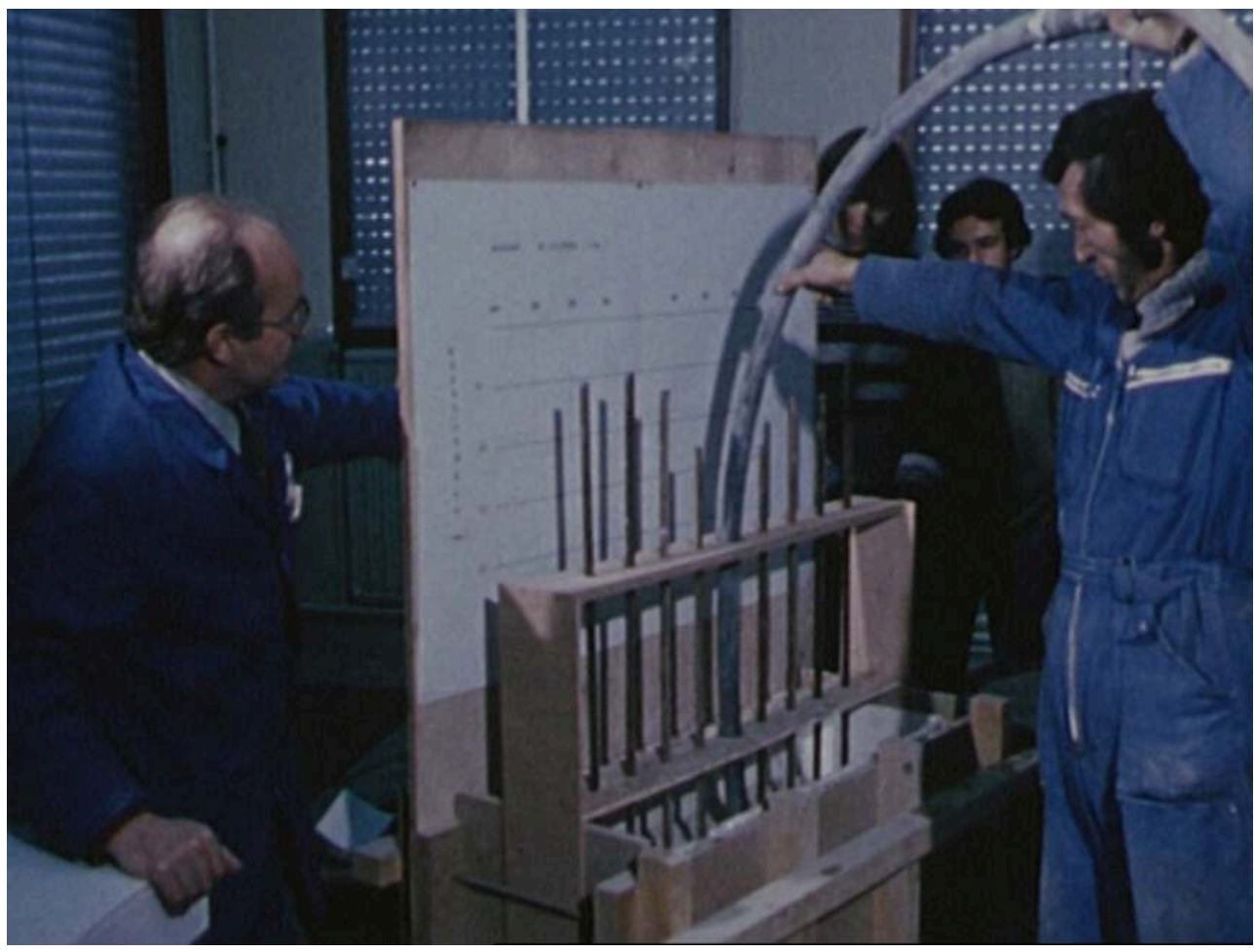

INA copie d'écran 25'52"

Figure 2 : mise en pratique de la vibration du béton au CET de Bagnolet

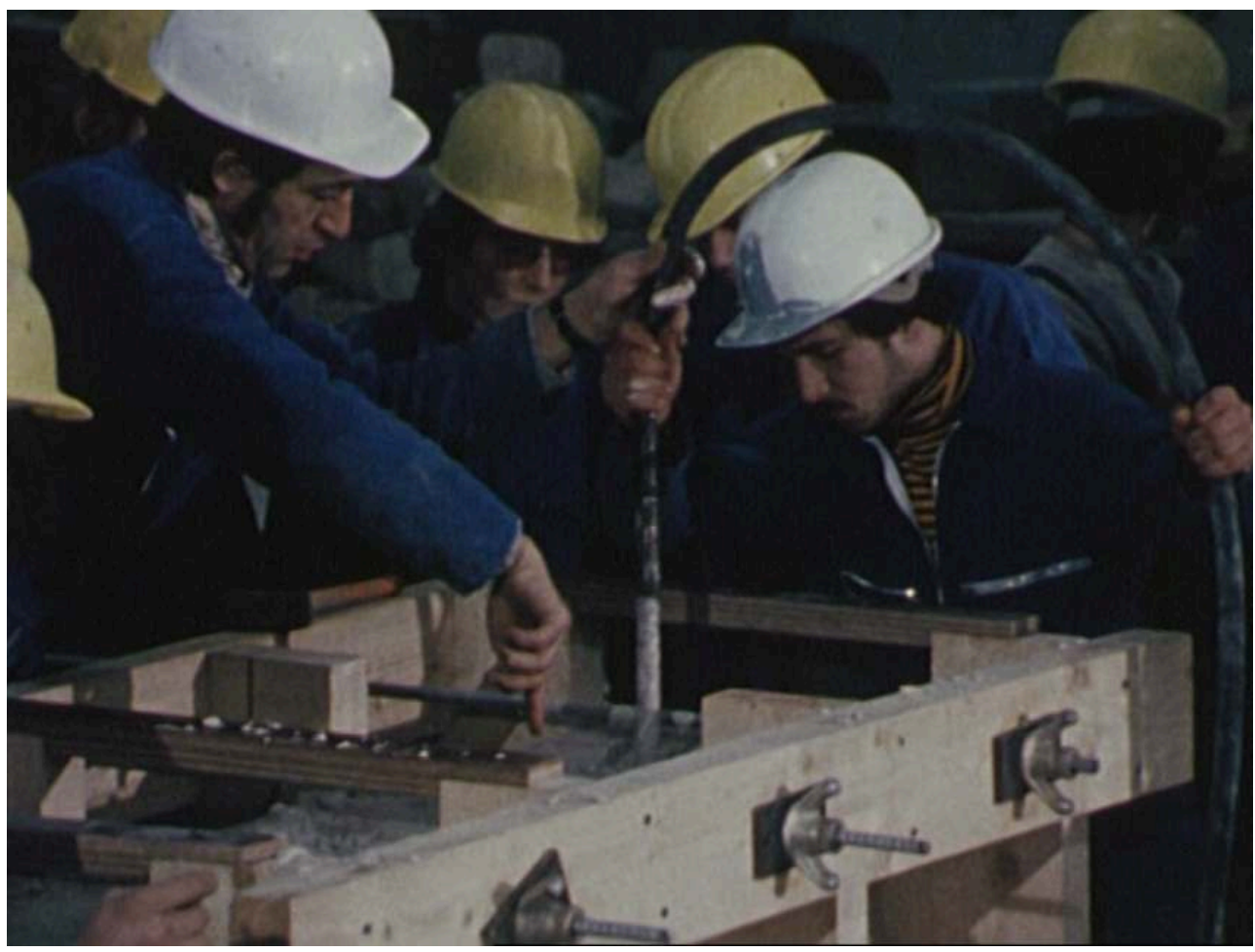

INA copie d'écran 27'33" 
Figure 3 : pose de l'acrotère produite par les élèves du CET de Bagnolet

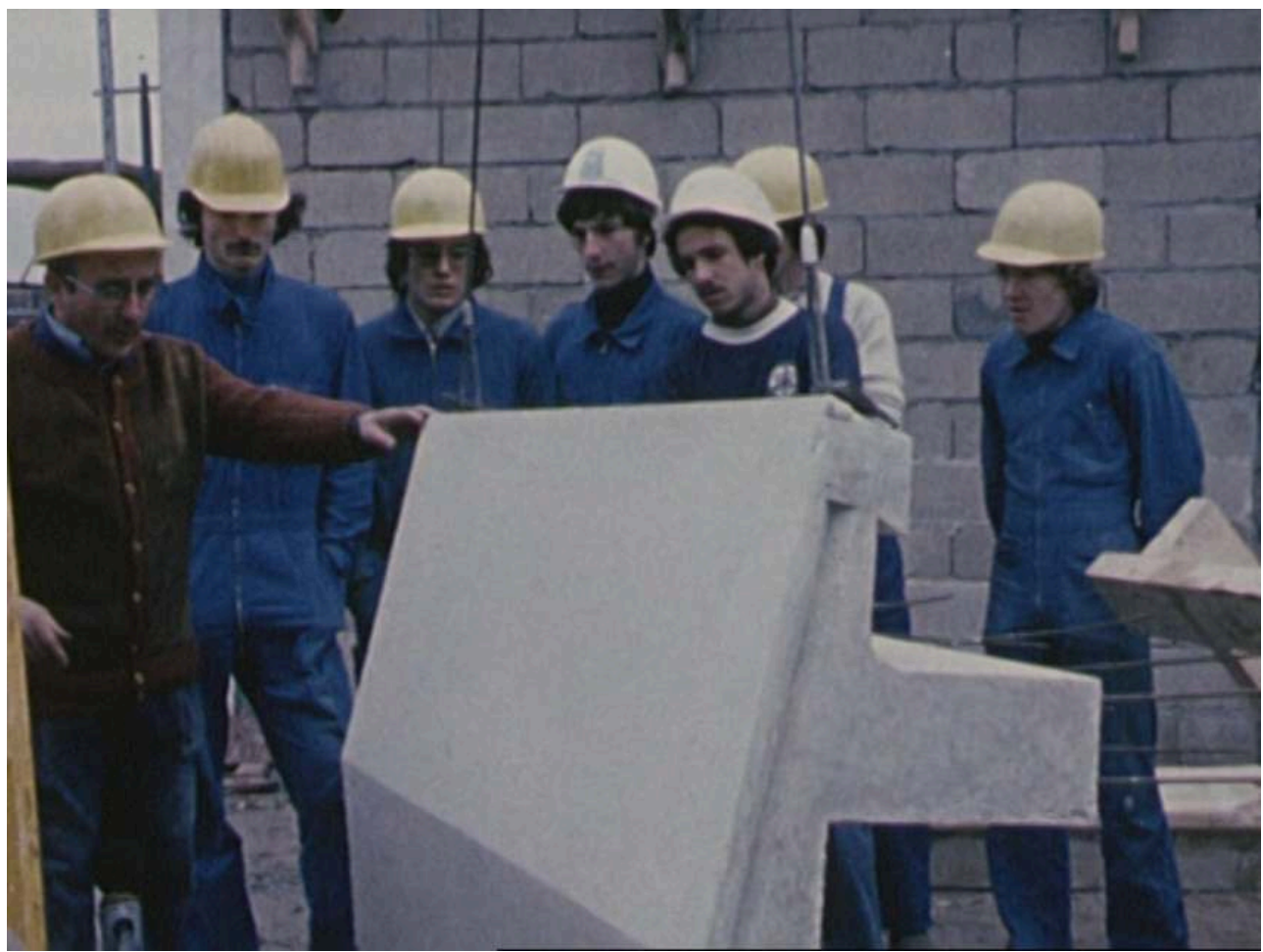

INA copie d'écran 28'08"

16 Au lycée d'Haubourdin, la conception et la fabrication d'un moule pour la production d'une pièce en plastique destinée à fixer les capotes des $2 \mathrm{CV}$ produites par Citroën sont présentées comme l'archétype de cette pédagogie de projet associant collectivement tous les enseignants et les élèves de l'établissement autour d'une réalisation concrète. La présentation des trente pièces constituant le moule produit par les élèves et les images $\mathrm{du}$ fonctionnement de la machine produisant les pièces plastiques viennent justifier le discours de Jean Pottier, qui, fort de cette pédagogie concrète, tend à nier les difficultés pédagogiques rencontrées par l'enseignement professionnel confronté à la massification, qui serait même avantagé sur le plan pédagogique par rapport à l'enseignement général.

«Dans les ateliers, nous n'avons pratiquement pas de problèmes de discipline. On a des problèmes de sécurité, bien sûr. On est souvent obligé d'intervenir. Mais nous avons très peu de problèmes de disciplines. Ils [les élèves] veulent faire et ils viennent demander eux-mêmes au professeur parce qu'ils veulent bien faire. Alors..., partant de là, nous pouvons dire que nous avons un avantage sur les professeurs des collèges qui n'ont qu'une craie et un tableau, même s'ils ont du matériel didactique, du matériel de laboratoire, c'est quand même un milieu artificiel. Nous, nous avons un milieu vraiment réel, tel que ces élèves souhaitent le trouver. (...) Quand on retourne à l'atelier, la réalité-là ne trompe pas. L'élève a immédiatement le résultat de ce qu'il a pensé, de ce qu'il a prévu, de ce qu'il a raisonné9. »

L'idée d'un enseignement professionnel comme voie de réussite pour les élèves ayant le moins d'appétence pour une culture scolaire abstraite est alors illustrée par un entretien entre un journaliste et un élève initialement en situation d'échec scolaire et orienté en fin de $5^{\mathrm{e}}$ vers un CAP de mécanicien, qui a obtenu un brevet de technicien en matière plastique avant d'intégrer une section de technicien supérieur. Mais, si 
l'illustration par l'image de la pédagogie active préconisée par l'institution scolaire apparaît comme l'argumentaire le plus efficace pour créditer l'enseignement technique et professionnel d'une capacité à préparer ses élèves à la vie active, d'autres aspects de cette communication institutionnelle sont très probablement d'une efficacité plus discutable.

\subsection{Un discours emphatique exaltant le métier et la technique}

Le projet des auteurs de La Réforme de l'éducation nationale de valorisation de l'enseignement professionnel et technique associe aux images des élèves au travail un commentaire destiné à donner à ces formations un prestige culturel comparable à l'enseignement du lycée général. La célèbre citation de Blaise Pascal, selon laquelle « La chose la plus importante à toute la vie est le choix du métier, le hasard en dispose ", est placée en exergue du documentaire sur l'enseignement professionnel. Puis, des références littéraires sont à plusieurs reprises mobilisées à cet effet dans le commentaire en voix off, ce qui n'est pas sans lui donner un aspect grandiloquent et sentencieux. Les images de la production de l'acrotère au CET de Bagnolet (fig. 3) sont placées sous les auspices de Paul Valéry pour qui le «travail est toute dépense d'actes qui tend à rendre les choses, les êtres, les circonstances profitables ou délectables à l'homme et l'homme lui-même plus sûr et plus fier de soi ». L'émerveillement de Guy de Maupassant lors d'une visite au Creusot, "royaume du fer où règne sa majesté le feu " d'après le texte publié dans Gil Blas le 28 août 1883, paraît bien éloigné des images d'une machine de production plasturgique que son évocation accompagne. Enfin, que dire du discours amphigourique associant des images du moule de la pièce de plasturgie produite au lycée d'Haubourdin tournant sur lui-même dans une ambiance irréelle sur fond de musique psychédélique, accompagné d'un commentaire évoquant la dimension artistique de la forme de cet objet en citant sur un ton affecté Terre des hommes de SaintExupéry?

La communication institutionnelle visant à défendre sans aucun contrepoint critique une politique gouvernementale atteint alors ses limites et souligne involontairement la domination culturelle de l'enseignement général. Cette survalorisation quelque peu forcée des filières de formation professionnelle et technique industrielle (Laurens $\mathrm{S}$. et Mischi J., 2011), dans cette France du milieu des années 1970 qui commence à découvrir la désindustrialisation et la réapparition d'un chômage massif, n'a probablement pas atteint pleinement son public. Il en est peut-être autrement dix ans plus tard sur la première chaîne de télévision lorsque l'émission Médiations met elle aussi particulièrement en lumière les filières industrielles de l'enseignement professionnel et technique pour évoquer la crise de certaines de ces formations.

\section{Médiations : la mise en scène d'une crise de l'enseignement professionnel?}

\subsection{Un enseignement professionnel associé à la notion d'échec scolaire}

En ouvrant la première partie de l'émission, intitulée " Témoignage ", par un reportage tourné dans un lycée professionnel (LP), les responsables de la ligne éditoriale de 
l'émission Médiations du 23 novembre 1987 associent étroitement l'enseignement professionnel et le terme d' "échec scolaire ", notion qui connaît une large diffusion tant dans les discours institutionnels que dans le champ médiatique dans les années 1980 (Ravon, 2000). Ce sujet d'une durée de près de six minutes, réalisé par Richard Michel et Jean-Marie Perthuis, propose au téléspectateur d'entrer dans le lycée d'enseignement professionnel Jules Michelet à Fontenay-sous-Bois (Val-de-Marne) dans une section industrielle où la formation apparaît particulièrement inadaptée aux réalités économiques des années 1980 et où les conditions d'enseignement sont particulièrement dégradées ${ }^{10}$.

21 L'association de l'enseignement professionnel à l'échec scolaire est explicite pendant la plus grande partie de la soirée. En effet, après la diffusion du reportage, les récits des élèves venant d'autres établissements présents en plateau insistent sur l'orientation par l'échec qui les a conduits vers l'enseignement professionnel et dont ils estiment avoir été victimes, ces nouveaux témoignages permettant une montée en généralité à partir de cet exemple.

Par rapport à La Réforme de l'éducation nationale, qui marquait dix ans plus tôt une entrée inédite des caméras dans les salles de classes et les ateliers, ce reportage est un exemple de l'approfondissement de cette évolution avec l'ouverture de l'institution scolaire à un journalisme audiovisuel indépendant des autorités publiques à partir du début des années 1980, sous l'effet d'un desserrement de la tutelle politique sur les rédactions des chaînes publiques et de l'apparition de télévisions privées. Sur le plan technique, cette évolution est rendue possible par le développement de moyens techniques plus légers de prise de vue. L'utilisation de caméra vidéo au format sur bande magnétique Betacam permet à une équipe légère de réaliser très rapidement des prises de vues et de son, y compris en extérieur, sans préparation particulière ${ }^{11}$.

Depuis l'échec de la politique d'unification du système scolaire sous le ministère d'Alain Savary en 1984, le discours médiatique apparait violemment hostile à l'enseignement public. L'enseignement professionnel, qui souffrirait d'un retard dans son adaptation au déclin des activités industrielles traditionnelles et parallèlement à l'accélération de la tertiarisation de l'économie, est particulièrement pris à partie pour expliquer les difficultés d'insertion dans l'emploi de la jeunesse. Par exemple, sur Antenne 2, le jeudi 12 septembre 1985, en seconde partie de soirée, Le Magazine diffuse un reportage sur l'enseignement professionnel à Saint-Nazaire intitulé "Impasse", qui accumule les récriminations contre cet enseignement, le LEP étant assimilé à une " voie de garage ", qui serait pour la plupart de ses élèves " synonyme d'échec »"

24 Aussi, le choix d'ouvrir une émission sur le thème de l'«échec scolaire " par un reportage tourné dans une section de mécanique générale de la proche banlieue de Paris en cours de désindustrialisation depuis les politiques de déconcentration des années 1960, s'inscrit-il dans la continuité de ce discours télévisuel du milieu des années 1980. Lorsqu'il annonce le reportage, le présentateur, François de Closets, signale bien que le lycée professionnel Michelet abrite dans sa partie la plus moderne, comme en attestent les images fugaces qui illustrent ce propos, une section dédiée à l'enseignement de l'informatique. Cependant, si l'animateur rappelle après la diffusion du reportage que la rénovation de la partie industrielle de l'établissement doit avoir lieu dans deux ans, il interroge immédiatement Hervé Hamon sur la représentativité de cet établissement, en créditant ce journaliste d'une connaissance générale de la situation de l'enseignement professionnel fondée sur la longue enquête de terrain 
menée avec Patrick Rotman pour la série de documentaires Tant qu'il y aura des profs diffusée trois ans plus tôt, en octobre 1984. Celui-ci confirme sans hésiter :

« On ne les a pas vus tous, on en a vu beaucoup et s'il y a une chose que je peux vous dire : c'est ça, c'est exactement ça, c'est exactement ce qu'on vient de voir. Dans un nombre considérable d'établissements, c'est tout ce qu'on vient de voir, c'est à dire : la décrépitude des locaux, mais c'est pas important, des machines-outils qui ne peuvent plus servir à rien, des formations désuètes, pointues, avec des enseignants qui sont enfermés dans des qualifications qui ne trouveront pas, de toute façon, de débouchés à l'extérieur, et surtout des enfants, vous l'avez entendu, qui ont totalement intériorisé leur échec, l'idée qu'ils sont fichus, complètement fichus, liquidés pour la société ${ }^{13}$.»

Cette association de la notion d'échec à l'enseignement professionnel est par la suite réitérée par Bertrand Girod de l'Ain, professeur de sciences de l'éducation à l'université Paris-Dauphine qui a joué un rôle important sur les questions éducatives dans le champ journalistique ${ }^{14}$. Il considère en effet sans nuance que, au mieux, seul le tiers des élèves qui obtient un baccalauréat général peut être considéré comme ayant réussi sa scolarité : « ceux qui ne sont pas là, c'est l'échec total ». En exposant le téléspectateur à la réalité très dégradée des formations industrielles dans certains établissements de cette époque, les images des élèves de la section de mécanique du lycée d'enseignement professionnel de Fontenay-sous-Bois ont aussi pour fonction de créditer cette critique d'un système éducatif vecteur d'échec scolaire massif, l'ensemble de l'enseignement professionnel et technique étant, dans un premier temps du moins, renvoyé à ce constat.

\subsection{Les forçats d'un enseignement industriel en déclin}

Le reportage commence à l'entrée du LP par une discussion animée avec les élèves de la section mécanique générale. Dix ans après la mise en œuvre à la rentrée 1977 de la loi du 11 juillet 1975 prévoyant la disparition progressive de ce palier d'orientation, ces jeunes appartiennent à la petite minorité, parmi les élèves les plus en difficulté, qui sont encore orientés précocement à ce stade de leur parcours scolaire. Leur nombre est d'ailleurs en très forte régression dans les années $1980^{15}$. Sans surprise, ils expriment leur amertume d'avoir été orientés si tôt vers une filière qu'aucun ne semble avoir choisie et dénoncent l'état de délabrement des installations et l'absence d'équipement de production moderne. L'un d'entre eux explique : « on aura un CAP et après quand on va aller travailler, ils vont nous présenter des machines avec des commandes numériques, on saura jamais nous en servir ${ }^{16}$. Ils expriment surtout le sentiment d'avoir en commun leurs parcours d'échec scolaire et l'établissement est désigné comme un "dépotoir », voire un "asile », tant sa réalité quotidienne apparaît hors du temps et en décalage avec le monde extérieur. Surtout, ces adolescents rejettent le travail qui leur est demandé, consistant à limer jour après jour des pièces en atelier. Si cette activité avait une valeur formatrice qui en faisait un enjeu de la formation de la main d'œuvre industrielle jusque dans les années 1950, elle est dès les années 1970 considérée comme le type même de l'« exercice-poubelle » (Pelpel, 2000) qui n'a plus d'autre fin et d'autre résultat que lui-même, ce dont les élèves sont parfaitement conscients: "avant ça marchait, on faisait de la lime. Mais maintenant c'est des machines qui font tout ça ». viennent corroborer leurs déclarations. La séquence tournée dans l'atelier révèle aussi 
le contraste entre la liberté de parole et de mouvement des élèves à l'extérieur et l'absurdité du travail réalisé à l'intérieur. Silencieux, ils sont filmés rivés à leur établi pour limer interminablement les même pièces métalliques, penchés sur leur travail sans que leur visage soit visible (fig. 4 et 5). Bien que l'activité des élèves dans l'atelier d'un LP ne puisse pas être considérée comme un travail strictement productif et n'a pas de rapport direct avec une organisation taylorienne de la production, les prises de vue captant la synchronie des mouvements que les élèves sont amenés à reproduire indéfiniment renvoient aux représentations stéréotypées d'un travail industriel aliénant dans lequel l'ouvrier, reconnaissable à son bleu de travail, courbé sur son ouvrage, a perdu la maîtrise du sens de son travail, représentations très éloignées de la réalité générale du travail industriel ${ }^{17}$.

Figure 4 : le travail à la lime des élèves du LP de Fontenay-sous-Bois (vue générale)

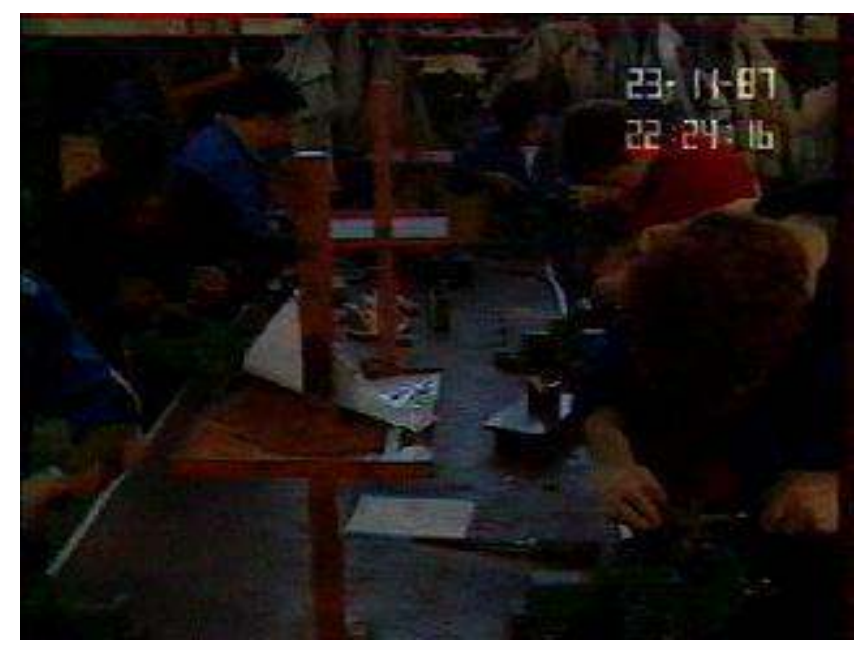

INA copie d'écran 22h24'16"

Figure 5 : le travail à la lime des élèves du LP de Fontenay-sous-Bois (gros plan)

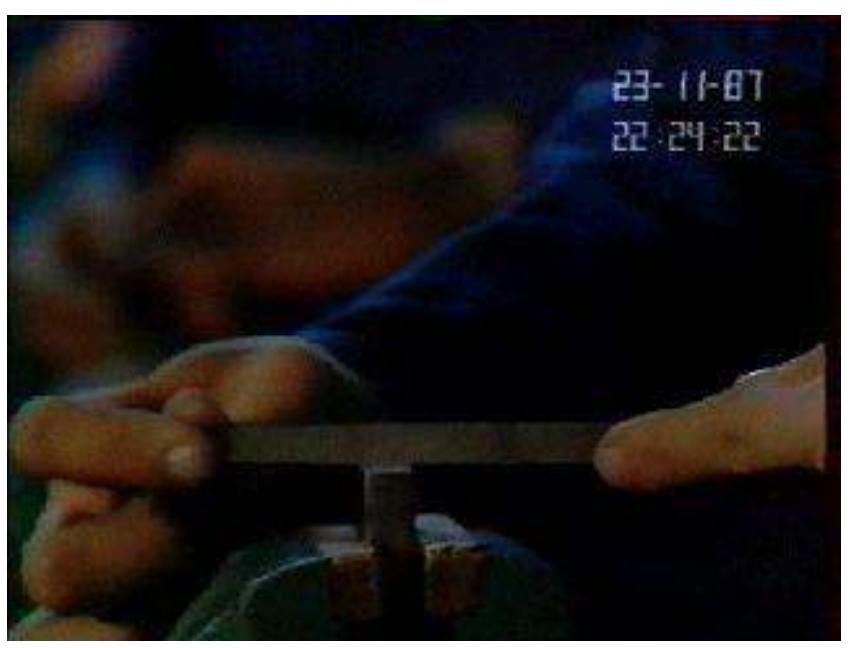

INA copie d'écran 22h24'22"

Le contraste est saisissant avec l'image que le documentaire dédié au "lycée demain » de la Réforme de l'éducation nationale voulait donner des formations industrielles dix ans plus tôt, par exemple en montrant les élèves s'affairer dans l'atelier de fabrication 
mécanique du lycée technique Jean Bart de Dunkerque (Nord) sur leur machine respective à différents stades de leur fonctionnement, comme le souligne le travelling latéral de la caméra donnant le spectacle d'une usine scolaire (fig.6).

Figure 6 : le travail dans l'atelier de mécanique du Lycée Jean Bart de Dunkerque

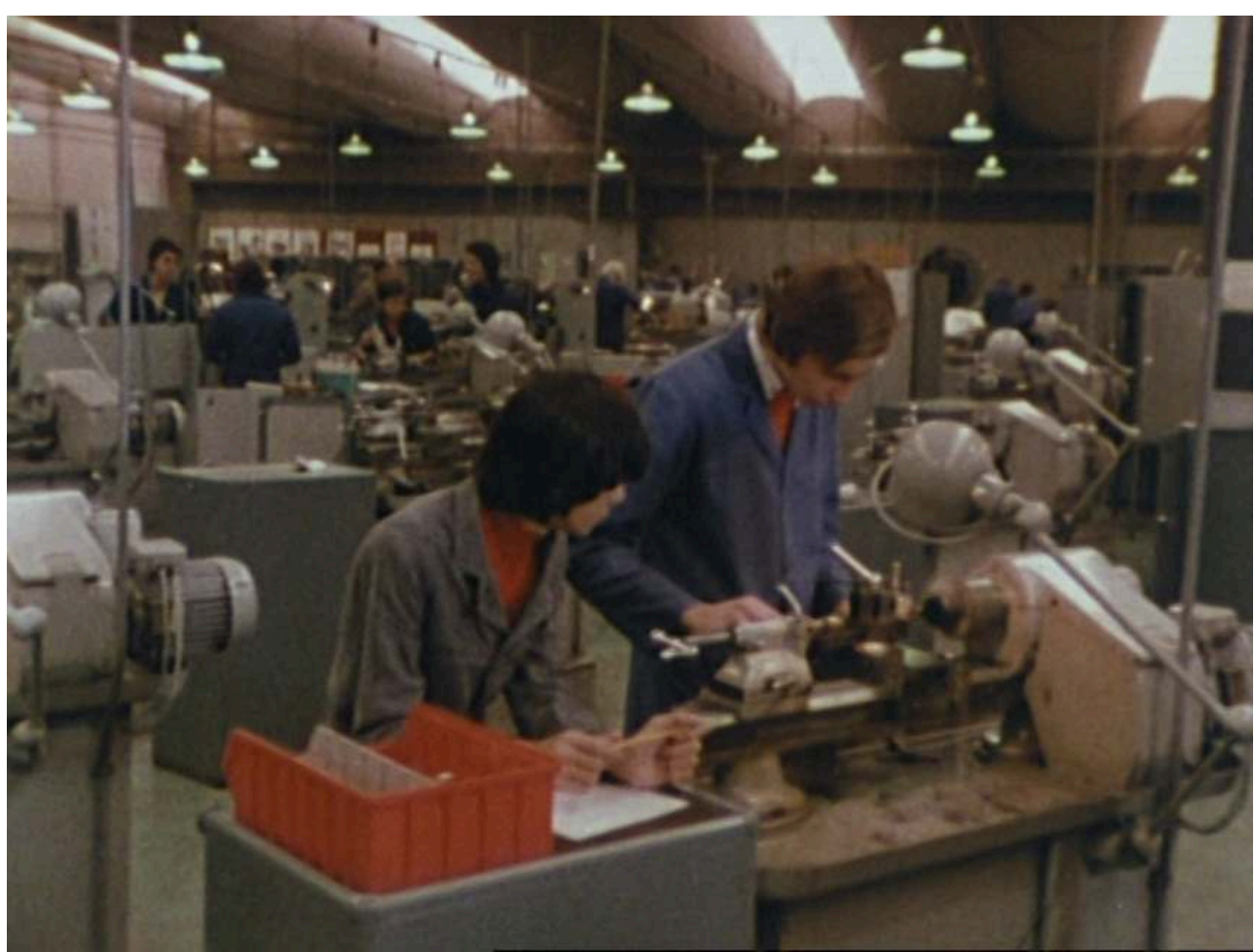

INA copie d'écran 7'15"

Enfin, à cette métaphore du déclin des industries traditionnelles dans la France des années 1980, s'ajoute la dissociation entre l'activité manuelle des élèves sur leur établi et les machines inertes entassées de l'autre côté de l'atelier, abandonnées en raison de leur obsolescence ${ }^{18}$.

Le trait est tellement accusé dans le reportage, puis dans la manière dont le dispositif de l'émission a permis de donner à l'exemple du LP Michelet un brevet de représentativité, que Jean-Pierre Chaudet, recteur de l'académie de Clermont-Ferrand, porte-parole de l'Éducation nationale sur le plateau, réclame inopinément la parole pour contester cette idée d'un enseignement professionnel déliquescent. Sans contester la réalité de la situation exposée dans le reportage, il insiste sur le fait que les lois de décentralisation auraient permis aux régions d'investir dans des machines à commande numérique, qui seraient présentes désormais dans la plupart des ateliers. Cette intervention assez véhémente oblige François de Closets à anticiper l'annonce d'un second reportage allant dans ce sens, qui sera diffusé dans la dernière partie de l'émission.

31 En définitive, le regard porté sur la réalité filmée à la fin des années 1970 comme à la fin des années 1980 est chargé d'une certaine représentation de l'enseignement professionnel industriel. D'abord vecteur d'une rénovation pédagogique promue par une institution publique dans une France pour laquelle l'industrie constitue un secteur d'activité essentiel à la pointe de la modernité, il devient le témoin de l'échec supposé 
du service public d'éducation à répondre aux défis des nouvelles mutations technologiques, au service d'une économie en cours de tertiarisation dans un contexte marqué par un changement de paradigme économique.

\section{Des images au service d'une inflexion idéologique libérale?}

\subsection{Insuffler un « esprit d'entreprise » dès le milieu des années 1970}

À la veille de la mise en œuvre de la loi du 11 juillet 1975, La Réforme de l'éducation nationale ne se contente pas de présenter à la population les principales dispositions des décrets d'application mis en œuvre à la rentrée 1977. À travers la présentation de pratiques innovantes choisies dans des établissements chargés de les expérimenter, ces films véhiculent des conceptions relevant d'une logique néo-libérale et constituent une des manifestations du changement de paradigme économique et social qui se manifeste à partir du milieu des années 1970, après plusieurs décennies marquées par le compromis salarial fordiste et la domination des conceptions macro-économiques keynésienne. En effet, la pédagogie de projet qui occupe une place centrale dans l'enseignement professionnel et technique tel qu'il est mis en image dans La Réforme de l'éducation nationale apparaît aussi comme une véritable pédagogie entrepreneuriale, qui témoigne d'une transformation des finalités de l'enseignement professionnel.

Dans Le lycée... demain, diffusé le 31 mai 1977, le projet de fabrication d'un écran de projection électrique facile à manipuler pour les enseignants conduit le lycée technique d'Aulnay-sous-Bois à se constituer en entreprise commerciale, avec pour résultat économique la commercialisation d'une cinquantaine d'écran à divers établissements scolaires. Le proviseur s'en félicite : «C'est la première fois que cette vente a eu lieu après un travail de relation avec le secteur commercial de l'établissement, après une étude de marché, après un travail de prospection à l'aide de documents publicitaires $»^{19}$. Cette activité serait donc une source d'émulation pour les élèves et les professeurs impliqués dans un projet mettant en relation le travail des différentes sections de l'établissement de la conception du projet à la commercialisation.

Le documentaire présente ainsi d'abord la fabrication des écrans, avant d'interroger une enseignante, qui explique que l'objectif était de créer un "esprit d'entreprise " dans le lycée, sur le rôle des sections tertiaires de l'établissement (fig. 7 à 9) : faire l'étude de marché, réaliser une brochure publicitaire, calculer le prix de revient, rédiger les courriers, concevoir et mettre à jour un fichier informatisé des fournisseurs. 
Figure 7 : production au lycée technique d'Aulnay-sous-Bois

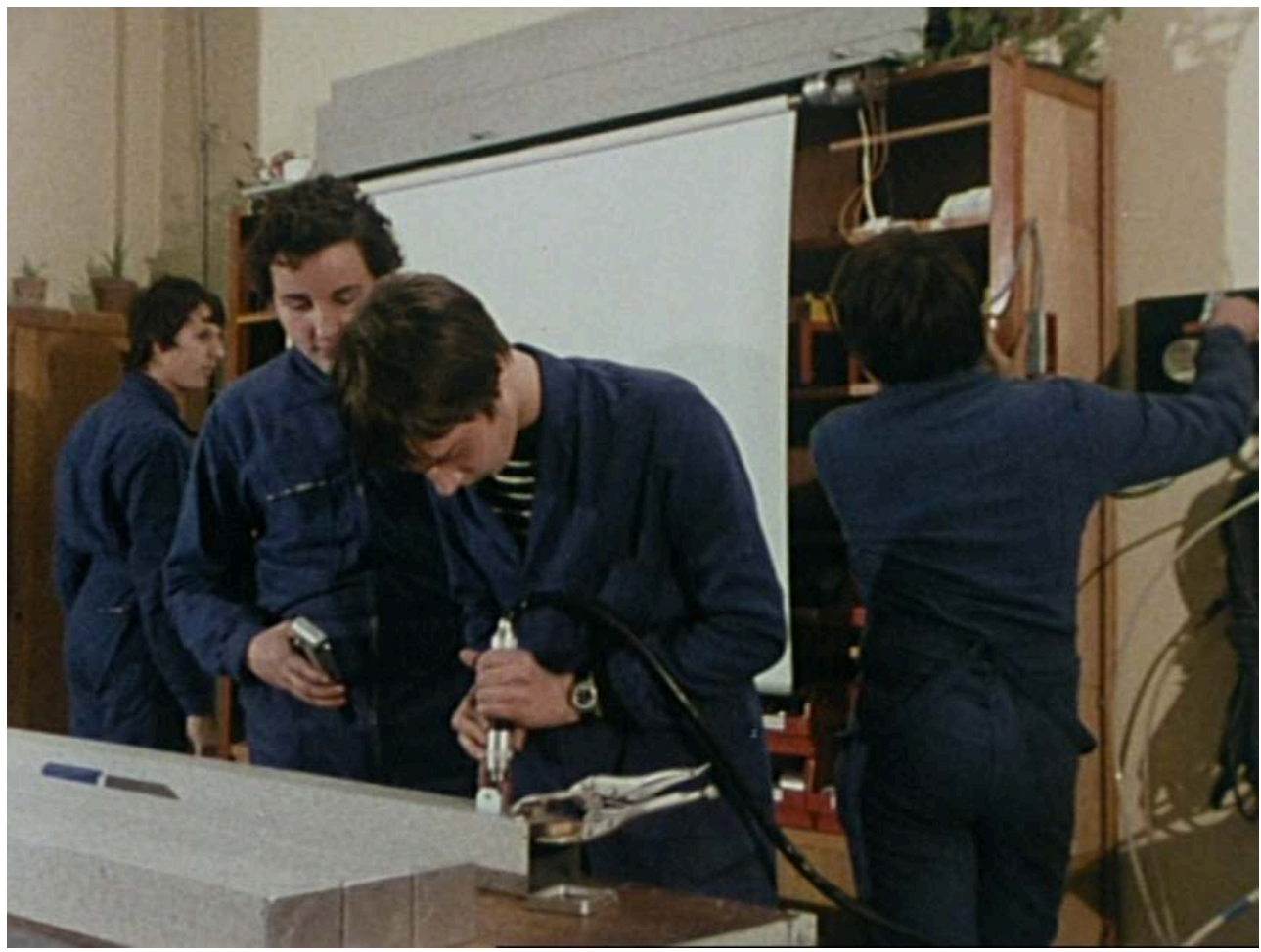

INA copie d'écran 45'30"

Figure 8 : secrétariat-comptabilité au lycée technique d'Aulnay-sous-Bois

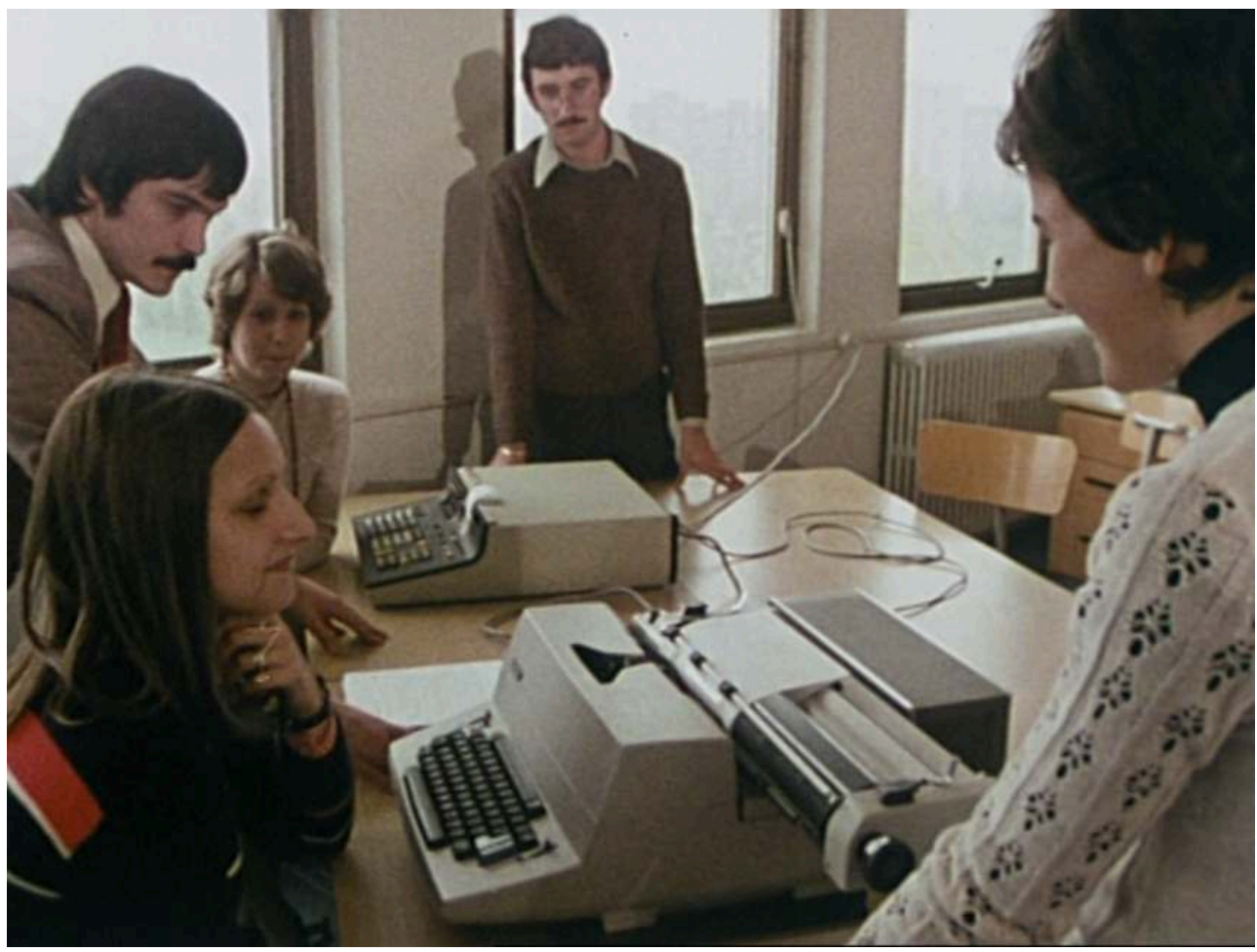

INA copie d'écran 48'49" 
Figure 9 : informatique au lycée technique d'Aulnay-sous-Bois

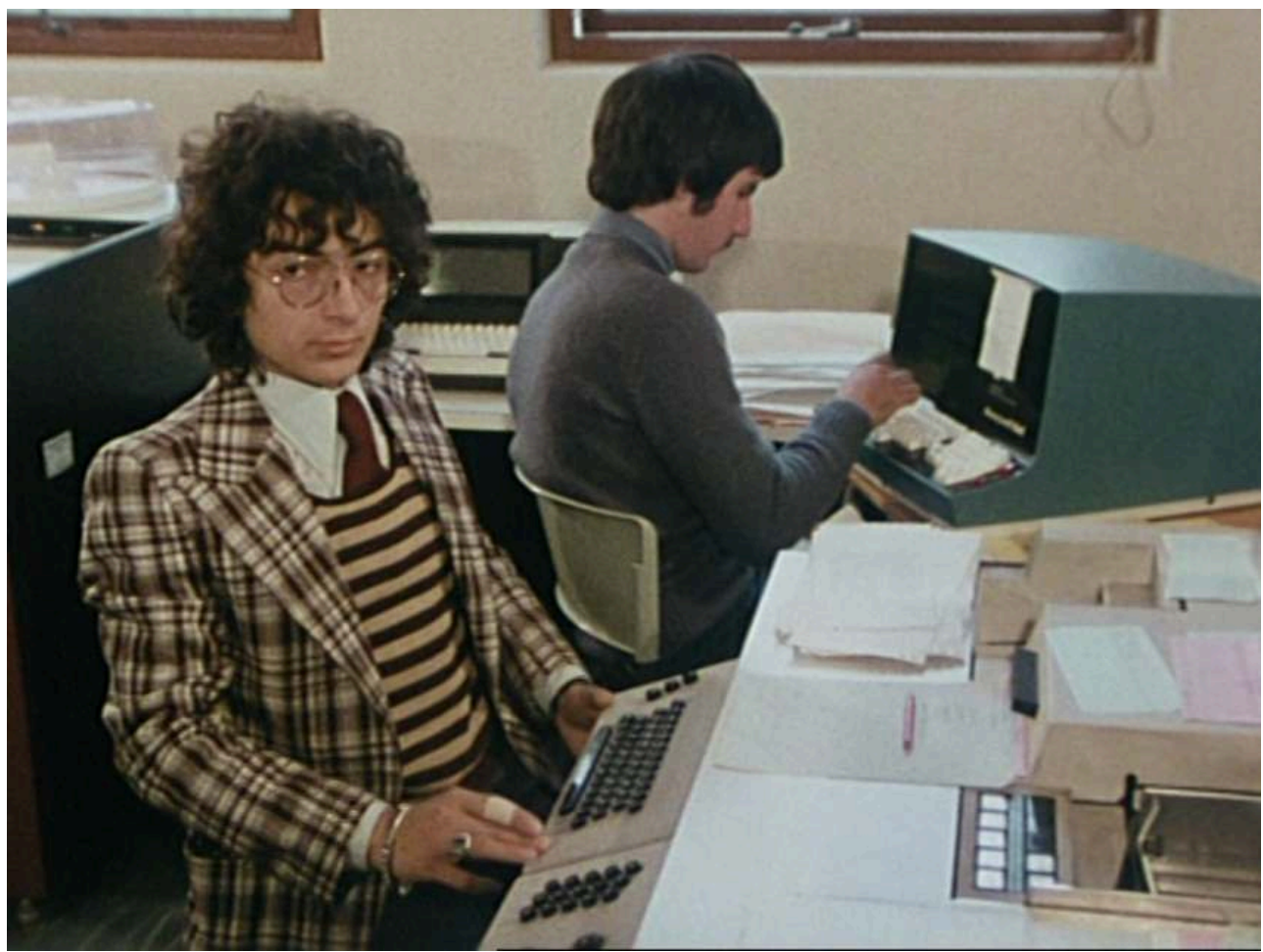

INA copie d'écran 49'55"

Seules les activités de production industrielle apparaissent dans le documentaire sur les LEP diffusé la semaine suivante. Néanmoins, la même démarche entrepreneuriale est présente à Haubourdin dans la conception et la fabrication du moule de la pièce en plastique servant à la fixation des capotes de $2 \mathrm{CV}$. En effet, le commentaire explique qu'aucun constructeur français ne pouvant ou voulant fabriquer cette pièce, le lycée se proposa d'assurer cette fabrication et gagna la commande en réalisant une authentique production industrielle de 2000 pièces par jour ${ }^{20}$, l'établissement public d'enseignement devenant sur le plan pratique une entreprise assurant une véritable production industrielle, sans que le cadre juridique de cette expérience ne soit précisé.

\subsection{Promouvoir un enseignement professionnel sous la tutelle exclusive des entreprises : le tournant des années 1980}

Dans la dernière partie de l'émission Médiations du 23 novembre 1987, le dernier reportage, que François de Closets a été obligé d'annoncer précocement, est construit comme le contrepoint du sujet réalisé au LEP Michelet de Fontenay-sous-Bois ${ }^{21}$. Il est censé donner à voir " un autre LEP ", le lycée Arbez-Carme situé à Oyonnax au cœur d'un bassin industriel dynamique. La présentation de cet établissement dédié à la plasturgie montre au téléspectateur des élèves motivés, vantant leur réussite personnelle, facilitée par des professeurs fortement impliqués dans une formation à la pointe du renouvellement technologique de ce secteur d'activité encore en plein essor $^{22}$. Les images des ateliers du lycée Arbez-Carme insistent particulièrement sur la modernité technologique des machines utilisées, notamment les machines à commande 
numérique, cruellement absentes pour les élèves et les professeurs à Fontenay-sousBois.

Figure 10 : travail sur une machine numérique dans l'atelier du lycée Arbez-Carme d'Oyonnax

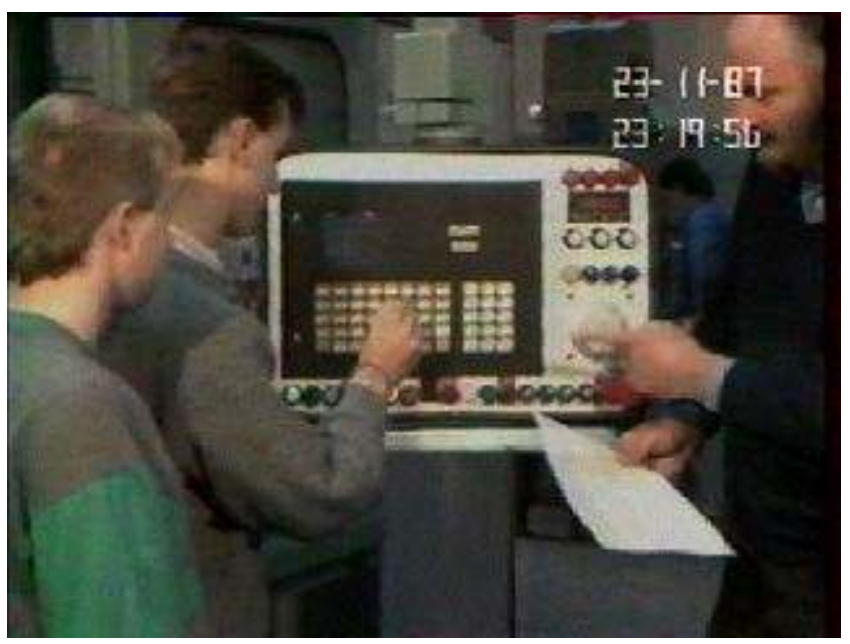

INA copie d'écran 23h19'56"

Les élèves sont systématiquement interrogés dans l'atelier, passant devant une machine parfois en cours de fonctionnement. Leur identité et leur situation scolaire sont systématiquement indiquées à l'écran alors que dans le précédent reportage leurs collègues de Fontenay-sous-Bois étaient collectivement et anonymement interrogés à l'extérieur de l'établissement. De plus, les élèves en activité visibles à l'écran sont postés sur des machines à commande numérique (fig. 10) mais surtout devant des ordinateurs (fig. 11), par exemple pour utiliser un logiciel de conception assistée par ordinateur grâce à une ardoise informatique ou pour suivre un programme d'initiation à un système d'exploitation informatique utilisés dans l'industrie dans les années 1980 . Ces images rompent avec l'aliénation physique des élèves de Fontenay-sous-Bois condamnés à la lime dans leur atelier de mécanique.

Figure 11 : L'utilisation de l'informatique au lycée Arbez-Carme d'Oyonnax

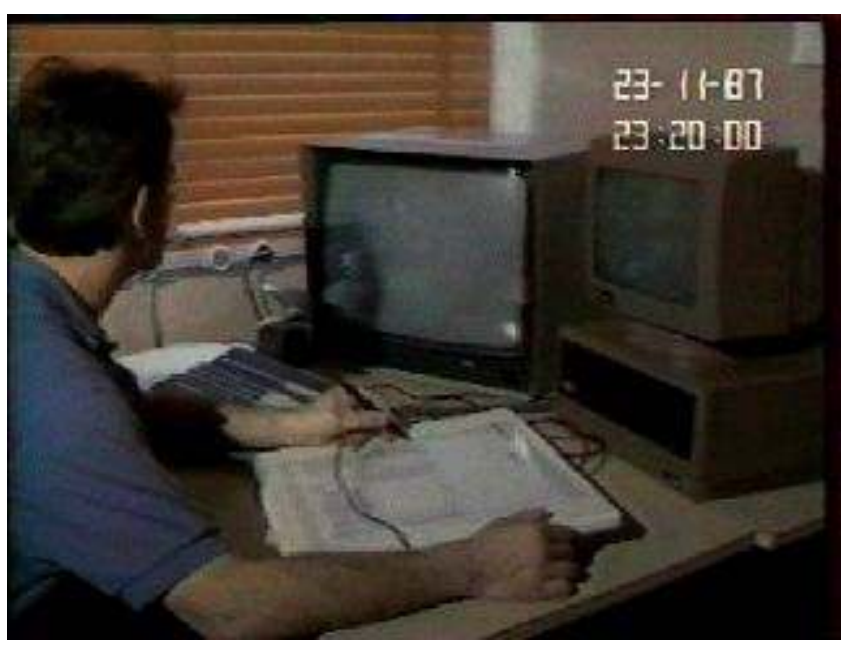

INA 23h20'00" 

principal facteur d'efficacité de la formation. Le lycée bénéficie du versement direct de la taxe d'apprentissage des entreprises locales. De plus, des fabricants de machines, qui ont compris l'intérêt qu'ils pouvaient avoir à ce que les futurs ouvriers et techniciens soient formés sur celles-ci, prêtent leur matériel au lycée. Enfin, les élèves peuvent effectuer des stages en entreprises, permettant par la même occasion à leur professeur de connaître les transformations du travail et de la production sur le terrain ${ }^{23}$. Ainsi, alors que, dans La Réforme de l'éducation nationale, le LEP devait mimer la vie économique de l'entreprise tout en restant clos sur lui-même, désormais la qualité de la formation est censée être proportionnelle à l'ampleur de l'intervention des entreprises dans la vie d'un établissement véritablement ouvert sur l'extérieur ${ }^{24}$.

Cette synergie apparaît comme une réalité nouvelle alors que la prise en considération de l'histoire particulière de cet établissement permettrait de mieux comprendre ce qui le différencie du LEP de Fontenay-sous-Bois. En effet, dans les années 1980, plus qu'un simple LEP, le lycée Arbez-Carme d'Oyonnax est alors un des fleurons de l'enseignement technique depuis plus d'un demi-siècle. École primaire supérieure transformée en école pratique de commerce en 1906, cet établissement se spécialise dans la plasturgie dès l'entre-deux-guerres et devient à ce titre en 1932 une des 32 écoles nationales professionnelles existantes. Ce sont deux professeurs de l'école qui conçoivent la première machine de moulage par injection entièrement automatique, à commande électromécanique, à la veille de la Seconde Guerre mondiale ${ }^{25}$. L'école devient un lycée technique d'État dès 1958. La comparaison de cet établissement regroupant des formations de niveau BEP, baccalauréat technologique et BTS dans un secteur de pointe avec une section de mécanique générale laissée à l'abandon à la veille de sa disparition programmée pour laisser place à une section conduisant à un CAP d'électricien, apparaît pour le moins orientée. Mais le premier reportage, qui a une valeur de témoignage sur la situation critique de certaines formations industrielles dans les années 1980, prend alors tout son sens dans la mesure où il permet de promouvoir sur la plateau des «solutions à l'échec scolaire " préconisées par Yvon Gattaz, intervenant en plateau en tant que président de l'association Jeunesse et Entreprise mais surtout connu comme président du Conseil national du patronat français de 1981 à 1986 (Daumas, 2010), et qui propose de «libéraliser totalement l'enseignement » en plaçant l'enseignement professionnel et technique sous la tutelle étroite des entreprises.

Les images télévisuelles de l'enseignement professionnel doivent donc bien être analysées sur le plan historique comme un élément d'un discours plus vaste qui se structure progressivement au cours de cette période charnière essentielle, du milieu des années 1970 à la fin des années 1980. Si, à travers La Réforme de l'éducation nationale, la télévision étatique assure la promotion d'une politique gouvernementale qui entend promouvoir massivement, sous la houlette des hauts fonctionnaires de l'Éducation nationale présents à l'écran, l'orientation des élèves vers un enseignement professionnel, les conceptions pédagogiques mises en avant sont déjà imprégnées des principes d'une nouvelle conception de la production et du management, en cours de généralisation en cette fin des années 1970, qui privilégie les notions de contrat et de projet. Dix ans plus tard, la première chaîne de télévision récemment privatisée entend promouvoir plus directement l'entreprise comme principal pilote de l'enseignement professionnel, quitte à rendre compte de façon parfois caricaturale des difficultés d'un 
enseignement professionnel pourtant en cours de rénovation et plus généralement d'un système scolaire transformé par d'ambitieuses politiques publiques de massification scolaire. Ce parti pris se manifeste notamment par la disparition des préoccupations pédagogiques dans un numéro de Médiations pourtant consacré à "l'échec scolaire». Aussi, dans l'attente d'une étude plus approfondie d'un corpus exhaustif de l'ensemble des émissions dédiés au système éducatif, la télévision apparaît bien, à travers les représentations de l'enseignement professionnel qu'elle véhicule, comme un des vecteurs de la popularisation de ce "nouvel esprit du capitalisme" (Boltanski et Chiapello, 1999), dont la domination a façonné la société française depuis cette époque, quelles que soient par ailleurs les réactions suscitées par la réception de ces discours.

\section{BIBLIOGRAPHIE}

Beaud S. (2000), «Le métier de chef de travaux : entre l'école et l'entreprise », Revue Française de pédagogie, $\mathrm{n}^{\circ} 131$, p. 23-31.

Boltanski L. et Chiapello E. (1999), Le nouvel esprit du capitalisme, Paris, Gallimard.

Brucy B. (2016), « la naissance du baccalauréat de technicien », in Fabienne Maillard et Stéphane Balas (coord.), Actes du colloque organisé pour les 30 ans du baccalauréat professionnel, CPC études, p. 193-204.

Chapoulie J.-M. (2010), L'École d'État conquiert la France. Deux siècles de politique scolaire, Rennes, Presses universitaires de Rennes, p. 479-482.

Daumas J.-C. (dir.), 2010, Dictionnaire historique des patrons français, Paris, Flammarion, p. 1128 et p. 1268.

Direction de l'Évaluation et de la Prospective (DEP) (1993), « Les transformations des scolarités au cours du cycle d'observation des collèges ", note d'information n93-36, Ministère de l'éducation nationale, p. 1 .

Faguer J.-P. (1983), « Le baccalauréat «E » et le mythe du technicien », Actes de la recherche en sciences sociales, vol. 50, p. 85-96.

Forestier Y. (2014), L'école, exception médiatique. La presse face aux enjeux des changements pédagogiques 1959-2008, thèse d'histoire contemporaine, université Paris IV, p. 96.

Gaillard I. (2012), La télévision. Histoire d'un objet de consommation (1945-1985), Paris/Bry-sur-Marne, CTHS/INA, p. 289.

Gutierrez L. et Legris P. (dir), 2016, Le Collège unique. Eclairages socio-historiques sur la loi du 11 juillet 1975, Rennes, Presses Universitaires de Rennes.

Jost F. (2007), Introduction à l'analyse de la télévision, Paris, Ellipses, p. 51-60.

Laurens S. et Mischi J. (2011), « Les politiques de « revalorisation du travail manuel » (1975-1981) », Agone, n46, p. 33-65.

Lembré, S. (2016), Histoire de l'enseignement technique, Paris, La découverte, p. 84-90. 
Palheta U. (2012), La domination scolaire, Paris, Presses universitaires de France.

Pelpel P. (2000), « Pratiques et modèles pédagogiques de l'enseignement technique », Revue française de Pédagogie, ${ }^{\circ} 131$, p. 48 et p.45.

Poels G. (2015), Les Trente Glorieuses du téléspectateur. Une histoire de la réception télévisuelle des années 1950 aux années 1980, Bry-sur-Marne, INA.

Ravon B. (2000), L'échec scolaire. Histoire d'un problème public, Paris, In Press éditions.

Rouquette S. (2002), Vie et mort des débats télévisés 1958-2000, Paris, De Boeck/INA, p. 134-135

Sauvage M. et Veyrat-Masson I. (2012), Histoire de la télévision de 1935 à nos jours, Paris, Nouveau Monde, p. 137 et p. 170.

Thévenot L. (1977), « Les catégories sociales en 1975. L'extension du salariat », Économie et statistiques, $\mathrm{n}^{\circ} 91$, p. 3-31.

Verret M. (1982), Le travail ouvrier, Paris, Armand Colin, p. 34.

\section{NOTES}

1. Maternelle, primaire, demain ", La Réforme de l'éducation nationale, 17 mai 1977, TF1, 20h30, durée : 50'03"; «Les collèges... », La Réforme de l'éducation nationale, 25 mai 1977, TF1, 20h30, durée : 54"51"; " Le lycée... demain », La Réforme de l'éducation nationale, 31 mai 1977, TF1, 20h30, durée : 54'38"; "Le lycée d'enseignement professionnel », La Réforme de l'éducation nationale, 7 juin 1977, TF1, 20h30, durée : 1h00'06».

2. L'échec scolaire », Médiations, 23 novembre 1987, TF1, 22h20, durée : 1h07'.

3. Les questions scolaires occupent une place très significative dans la collection de Médiations. Parmi les 42 numéros de Médiations, deux autres émissions sont consacrées au surmenage des élèves face à des programmes qui seraient surchargés et aux difficultés des élèves à s'orienter dans le second cycle du second degré. INA, «Le gavage des élèves », Médiations, 27 février 1989, TF1, 22h31, durée : 1h07'. INA, «Le pièges du bac », Médiations, 28 mai 1990, TF1, 22h31, durée : 1 h07'.

4. Les collèges... ", La Réforme de l'éducation nationale, op.cit., de 23'17 à 33'04".

5. Le choix de donner une telle place à une filière ne produisant qu'environ 6000 bacheliers par an à cette époque s'explique par la possibilité de promouvoir ainsi les lycées techniques en mettant en valeur l'élite de ses élèves. Celle-ci, tout en évoluant dans cet univers visuel de l'atelier, suit une formation qui s'apparente aux filières générales du lycée par l'ampleur de ses enseignements théoriques et par l'absence de débouché professionnel direct sans fréquentation de l'enseignement supérieur (Faguer, 1983).

6. Le lycée d'enseignement professionnel ", La Réforme de l'éducation nationale, op. cit., de 58'18 à $58^{\prime} 40$ ".

7. Ibid., de 12'59" à 14'04". Même si la part des ouvriers dans la population active a commencé à baisser dans les années 1960, le nombre d'ouvriers atteint en France son apogée en 1975 (Thévenot, 1977).

8. Le lycée... demain ", La Réforme de l'éducation nationale, op. cit., de 42'20 à 43'34".

9. le lycée enseignement professionnel ", La Réforme de l'éducation nationale, op. cit., de 43'34" à $45^{\prime} 15^{\prime \prime}$.

10. L'échec scolaire ", Médiations, op. cit., 22h22'05" à 22h27'56'.

11. Le premier format Betacam est apparu en 1983, suivi d'une version plus performante en 1987, le Betacam SP, qui devient très vite le standard de la plupart des chaînes de télévision jusqu'à la fin des années 1990. 
12. Impasse ", Le Magazine, 12 septembre 1985, Antenne 2, 23h25'31", durée : 8'01".

13. L'échec scolaire ", Médiations, op. cit., de 22h28'18" à 22h28'51".

14. Bertrand Girod de l'Ain a été la figure tutélaire de la rubrique éducation du journal Le Monde dans les années 1960. Secrétaire général de l'Association d'étude pour l'expansion de la recherche scientifique (AEERS), il a rédigé avec Louis Legrand l'un des rapports préparatoires au colloque réformateur d'Amiens en 1968. Il a quitté le journalisme en 1970 pour enseigner les sciences de l'éducation à Paris-Dauphine (Forestier, 2014).

15. Alors que $16 \%$ des élèves entrés en $6^{\mathrm{e}}$ en 1980 sont orientés deux ans plus tard vers la préparation d'un CAP dans un lycée d'enseignement professionnel, ce n'est plus le cas que de $4 \%$ de ceux entrés en $6^{\mathrm{e}}$ en 1989. Le palier d'orientation de fin de $5^{\mathrm{e}}$ est totalement supprimé en 1994 (DEP, 1993).

16. L'échec scolaire ", Médiations, op. cit., 22h25'05" à 22h25"13".

17. À la fin des années 1970 , on peut évaluer à $8 \%$ la part des ouvriers travaillant à la chaîne contre $32 \%$ qui sont postés sur des machines automatisées (Verret, 1982).

18. Un des deux journalistes qui interrogent $M$. Braunmeyer, le chef de travaux du lycée professionnel Michelet, lui fait remarquer que la machine placée devant porte une plaque datée de 1957. « L'échec scolaire ", Médiations, op. cit., 22h25'14" à 22h25'18".

19. Le lycée... demain ", La Réforme de l'éducation nationale, op. cit., de 45'38" à 55'55".

20. Le lycée d'enseignement professionnel ", La Réforme de l'éducation nationale, op. cit., de 33'15" à 34'02".

21. L'échec scolaire ", Médiations, op. cit., 23h18'15" à 22h21'28".

22. Dans La réforme de l'éducation nationale, à travers l'exemple du lycée professionnel Beaupré à Haubourdin, la plasturgie apparaît aussi comme l'exemple de l'activité incarnant la modernité industrielle. «Le lycée d'enseignement professionnel », La Réforme de l'éducation nationale, op. cit., de 32'29" à 33'14" et de 48'37" à 49'05". "L'échec scolaire ", Médiations, op. cit., 23h18'45" à 22h18'50". À dix ans d'intervalle, on retrouve les mêmes images de la coulée de la matière plastique en fusion qui fait référence dans les représentations collectives à la métallurgie triomphante de l'âge industriel du XIX ${ }^{\mathrm{e}}$ siècle.

23. Encore objet de débats animés au milieu des années 1970, l'introduction de stage en entreprise est justement généralisée par la création des baccalauréats professionnels au milieu des années 1980 dont les premiers diplômés viennent de sortir de l'enseignement professionnel à l'été 1987. Néanmoins, l'impossibilité pour les élèves de Fontenay-sous-Bois de faire des stages, soulignée dans le premier reportage, tend à faire de la situation au lycée Arbez-Carme d'Oyonnax un fait exceptionnel.

24. Stéphane Beaud a souligné le risque de perte d'autonomie de l'enseignement professionnel à propos de l'évolution du métier de chef de travaux dans les LP à cette époque (Beaud, 2000).

25. Selon le site internet de l'établissement : http://www.arbez-carme.com/2013-02-21-09-47-45/ son-histoire. Consulté le 17 juillet 2018.

\section{RÉSUMÉS}

Alors que l'essor de la télévision constitue un fait culturel majeur, la dynamique tant démographique, économique, sociale que culturelle de la France de l'après-guerre, qui se prolonge bien au-delà du milieu des années 1970 sur le plan scolaire, conduit à la mise en œuvre 
de politiques transformant profondément la place de l'enseignement professionnel au sein du système éducatif. Comment le discours télévisuel en a-t-il rendu compte pendant cette période charnière des années 1970-1980 qui connaît un profond changement du paradigme économique et social dominant? L'étude des archives audiovisuelles conservées par l'institut national de l'audiovisuel (INA) montre de quelle manière la question de l'enseignement professionnel est intégrée à un discours plus vaste, entre dénonciation des pesanteurs supposées du système éducatif et l'exaltation de l'œuvre modernisatrice en cours, alors même que le champ médiatique est profondément bouleversé par la libéralisation du secteur audiovisuel.

While the rise of television is a major cultural fact, the demographic, economic, social and cultural dynamics of post-war France, which extends well beyond the mid-1970s in the school fiels, leads to the implementation of policies that profoundly transform the place of vocational education within the education system. How did the televisual discourse account for it during this pivotal period of the 1970s-1980s, which is undergoing a profound change in the dominant economic and social paradigm? The study of audiovisual archives kept by the National Audiovisual Institute (INA) shows how the question of vocational education is integrated into a wider discourse, between denouncing the supposed gravities of the education system and exaltation of the modernizing work in progress, even as the media field is profoundly upset by the liberalization of the audiovisual sector.

\section{INDEX}

Mots-clés : enseignement professionnel, télévision, représentation, élève, orientation, politique scolaire

Keywords : professional education, television, representation, student, orientation, school policy

\section{AUTEUR \\ JÉRÔME KROP}

Maître de conférences en histoire contemporaine à l'université de Lille (Institut national supérieur du professorat et de l'éducation) et membre du laboratoire CREHS (université d'Artois). Après avoir étudié, dans le cadre de sa thèse, l'histoire de la première génération des instituteurs et institutrices de l'école républicaine dans Les fondateurs de l'école républicaine (Villeneuve d'Ascq, Presses universitaires du Septentrion, 2016) et interrogé le modèle scolaire méritocratique de la IIIe république (La méritocratie républicaine. Scolarisation de masse et élitisme scolaire sous la IIIe République, Rennes, Presses universitaires de Rennes, 2014), il s'intéresse depuis plus particulièrement la manière dont la société française a appréhendé la massification de l'enseignement secondaire entre 1958 et 1995. Il mène notamment un travail de recherche reposant sur l'exploitation des archives télévisuelles conservées par l'institut national de l'audiovisuel (INA) dans la perspective d'une histoire des représentations de la massification scolaire et de la place de la télévision dans les débats éducatifs. 